\title{
CFD BASED SIMULATION OF HYDROGEN RELEASE THROUGH ELLIPTICAL ORIFICES
}

\author{
Nasim Shishehgaran ${ }^{1}$, Marius Paraschivoiu ${ }^{1} *$ \\ ${ }^{I}$ Department of Mechanical and Industrial Engineering, Concordia University, 1455 De Maisonneuve \\ Blvd. West, Montreal, Quebec, H3G1M8, Canada \\ * Corresponding author \\ marius.paraschiviou@concordia.ca
}

Tel 1-514-848-2424 ext. 3147

Fax 1-514-848-3175

\begin{abstract}
Computational Fluid Dynamics (CFD) is employed to investigate the hydrogen jet exiting through different shapes of orifices. The effect of orifice geometry on the structure, development and dispersion of a highly under-expanded hydrogen jet close to the exit is numerically investigated. Various shapes of orifices are evaluated, including holes with constant areas such as elliptical and circular openings, as well as, enlarging circular orifices. A three-dimensional in-house parallel code is exploited to simulate the flow using an unstructured tetrahedral finite volume Euler solver. The numerical simulations indicate that, for a high pressure reservoir hydrogen release, the area of the orifice is the main geometric parameter influencing the centerline pressure at the hydrogen-air interface and the transient peak temperature, while the elliptical or expanding orifices slightly mitigate the auto-ignition risks associated with the accidental release of hydrogen. Therefore, circular openings represent the most conservative geometry for the study of auto-ignition of hydrogen.
\end{abstract}

Keywords: Hydrogen; Computational Fluid Dynamics; Under-expanded Jet; Elliptical Orifice; Enlarging Orifice

\subsection{INTRODUCTION}

The accidental release of hydrogen from a high-pressure reservoir into the low-pressure surrounding results in the formation of the under-expanded jet and can lead to auto-ignition or to the dispersion of a hydrogen cloud. Investigations of hydrogen dispersion are reported in various numerical and experimental studies [1-3]. Some studies have focused on the calculation of the hydrogen concentration and its relation to dimensions of the circular exit [1] [2]. Han et al. [4] studied the concentration distribution and the mass flux of hydrogen released from a pressurized tank through the different hole sizes (less than $1 \mathrm{~mm}$ ) and storage pressures (less than $400 \mathrm{bar}$ ). They defined the dilution length of a specific hydrogen mole fraction as a distance from the hole. Results represented a consistent decrease of the centerline hydrogen concentration and the dependency of mass reduction ratio on 1/d for different storage pressures less than 400 bar.

Other studies have focused on the evaluation of the ignition and the auto-ignition possibility of the hydrogen release. Radulescu et al. [5-6], analyzed the effect of the volumetric expansion on the ignition of high-pressure hydrogen release in a diffusion layer by applying the Lagrangian unsteady diffusion-reaction model. It was demonstrated that the strong expansion can lessen the ignition possibility. Furthermore, it was shown that for each storage pressure there exists a size of the hole that separates the non-ignition region from the auto-ignition region. Many studies have been conducted to 
clarify the effects of tube length and tube diameter on the auto-ignition possibility of the high-pressure hydrogen release [7-10]. Golub V. et al. [7] investigated the hydrogen auto-ignition in tubes both numerically and experimentally. The effect of the shape of the release tube on the auto-ignition was analyzed. Two different shapes of the tube were considered, circular and rectangular. It was shown that the auto-ignition in a rectangular tube occurred at a lower pressure compared to its equivalent circular tube.

Yamada E. et al. [9-10] carried out a direct numerical simulation (DNS) with a detailed chemical model to study the ignition of high-pressure hydrogen (40 MPa) discharging into the air. For a given tube diameter, the effect of varying tube lengths was evaluated. It was found that there is a relation between the length of the tube and the auto-ignition. A longer tube provides enough space for hydrogen and air to mix before the exit, and in turn, a higher possibility of auto-ignition. Very detailed direct numerical simulation DNS, which is made for smaller release pressures and longer release tubes, lead to similar conclusions [11-12]. Furthermore, it was recognized that longer tubes increase the ignition probability and for a higher release pressure, an auto-ignition can occur inside the release tube. This effect is related to the better mixing of hydrogen and air through molecular diffusion. Khaksarfard R. et al. [13] numerically investigated the expanding of the release area with different expansion rates. It was shown that the pressure on the contact surface is sensitive to the opening speed. The higher the opening speed, the higher the expansion of the hydrogen jet.

Most of the studies about hydrogen safety issues have focused on circular nozzles and the development of a hydrogen jet exiting from a standard round exit hole. Thus, owing to the lack of study of the hydrogen dispersion and its auto-ignition possibility under different conditions in terms of geometrical layout, this work aims to numerically investigate the effect of the orifice geometry on the behavior and development of the hydrogen jet issuing from different types of release exits. The novelty of this work is that various types of exits including elliptical, expanding and circular are considered, and jet characteristics and expansion are compared between these different cases.

\subsection{COMPUTATIONAL DOMAIN AND MODEL}

The 3D computational domain consists of a circular cylinder as a reservoir containing hydrogen gas at a high pressure and a $2 \mathrm{~mm}$ straight nozzle leading to the exit at the ambient pressure. To examine the influence of the orifice geometry on the jet behavior and the ignition possibility, three different shapes of orifices are considered including fixed circular, fixed elliptic and an expanding exits.

\subsection{Computational Model}

In this study, a three-dimensional in-house parallel code is exploited to simulate the flow. This code has been extensively validated with experimental results for a wide variety of CFD problems [14]. Abrupt discharging of hydrogen from a high-pressure tank into a low-pressure quiescent environment causes a highly under-expanded jet. Due to the high Reynolds number in the vicinity of the release hole, convection dominates over viscosity and diffusivity and the flow is modeled by the compressible Euler equations [3] [6].

$\frac{\partial \vec{U}}{\partial t}+\vec{\nabla} \cdot \overrightarrow{\vec{F}}(U)=0$,

Since a dynamic mesh algorithm based on the spring analogy is employed to simulate the enlarging orifices, the Euler equations must be modified. This method was described in [13]. Considering the dynamic mesh formulation in which the convection velocity components are the relative velocity between fluid and coordinates for a time-dependent system, the modified conservative fluxes in the Euler equations will be as follows: 


$$
\overrightarrow{\vec{F}}=\left(\left[\begin{array}{c}
\rho\left(u-w_{x}\right) \\
\rho\left(u-w_{x}\right) u+P \\
\rho\left(u-w_{x}\right) v \\
\rho\left(u-w_{x}\right) w \\
\rho\left(u-w_{x}\right) E+u P
\end{array}\right]\left[\begin{array}{c}
\rho\left(v-w_{y}\right) \\
\rho\left(v-w_{y}\right) u \\
\rho\left(v-w_{y}\right) v+P \\
\rho\left(v-w_{y}\right) w \\
\rho\left(v-w_{y}\right) E+v P
\end{array}\right]\left[\begin{array}{c}
\rho\left(w-w_{z}\right) \\
\rho\left(w-w_{z}\right) u \\
\rho\left(w-w_{z}\right) v \\
\rho\left(w-w_{z}\right) w+P \\
\rho\left(w-w_{z}\right) E+w_{P}
\end{array}\right]\right)
$$

Where $w_{x}, w_{y}, w_{z}$ are the grid speeds along the coordinate directions. In the case of a fixed mesh, the grid velocities are not considered and the cell volume is not time-dependent. A discrete form of the Euler equations based on the implicit finite volume method can be written as:

$\left|V_{i}\right| \frac{\vec{U}_{i}^{n+1}-\vec{U}_{i}^{n}}{\Delta t}+\sum_{\text {over } \partial V_{i}} \overrightarrow{\vec{F}}_{\partial V_{i}}^{n+1} \cdot \vec{n}_{\partial V_{i}} \Delta S_{\partial V_{i}}=0$

The system of linear equations is solved by means of a fully implicit scheme which has an accuracy of the first and second orders in time and space, respectively. Convective fluxes are evaluated using the Roe-MUSCLE scheme. To avoid the oscillations generated near the shock or discontinuity regions, the Van Leer-Van Albada limiter is used to control the accuracy of special approximation at the thin layer near the exit hole where the second-order approximation is switched to the first-order accuracy to prohibit the numerical instabilities in this region.

The system of Euler equations is completed by the Abel Nobel equation of state which is defined as follows [11][13]:

$p=\frac{R_{\text {mix }} T}{(v-b)}=(1-b \rho)^{-1} \rho R_{\text {mix }} T=\zeta \rho R_{\text {mix }} T, \quad b=0.00775 \mathrm{~m}^{3} / \mathrm{kg}$

Then the system of equations is solved using an iterative GMRES solver and MPI parallel processing.

To describe the convection of hydrogen into the ambient air and track the shape and the location of the contact surface between hydrogen and air, the advection equation is implemented. The modified form of the transport equation for the dynamic mesh model is written as follows:

$\frac{\partial c}{\partial t}+\frac{\partial\left(c\left(u-w_{x}\right)\right)}{\partial x}+\frac{\partial\left(c\left(v-w_{y}\right)\right)}{\partial y}+\frac{\partial\left(c\left(w-w_{z}\right)\right)}{\partial z}=0$

Where $c$ is a fraction function. A unit value of the fraction function $(c=1)$ corresponds to a cell occupied only by air, and a zero value $(\mathrm{c}=0)$ indicates a cell full of hydrogen. The fraction functions between zero and one $(0<\mathrm{c}<1)$ indicate the discontinuity region and show the cell contains the interface between hydrogen and air. After calculating $\mathrm{c}$, the average value of the gas constant, $R$, for the hydrogen-air mixture is solved by equation (4).

$R=R_{H_{2}}(1-c)+R_{\text {air }} c$

Where $R_{\mathrm{H}_{2}}=4124 \mathrm{~J} / \mathrm{kgK}$ and $R_{\text {air }}=287 \mathrm{~J} / \mathrm{kgK}$. The specific heats are assumed to be constant throughout the release, thus, $\gamma=1.4$.

\subsection{Computational Domain}

The mathematical domain is discretized into an unstructured tetrahedral mesh. The discretized domain contains almost 2 million nodes and 12 million tetrahedrons. To ensure that the mesh is sufficiently refined to resolve large pressure gradients and all relevant flow features in the proximity of the exit area, a very fine grid resolution is generated in this region. Then the element size increases with a growth rate of 1.02 downstream and in the regions far from the exit. This mesh was tested for mesh independency in [15] so that additional refinement of the mesh does not change the reported results. 
The METIS software package is used to distribute the mesh elements to the processors and partition the domain for parallel computing.

The viscosity effect and the heat transfer between the gas inside the reservoir and its surrounding are neglected; therefore all the solid walls of the high-pressure tank and the release tube are assumed to be slip and adiabatic. The non-reflecting far field boundary condition was applied around the circumference of the low-pressure cylinder (external environment) and at the end of the cylinder. It is supposed that there are no external forces. Hence, the effect of the gravity on the fluid is neglected.

The flow is initially at rest with zero velocity. The reservoir and the half of the tube are filled with hydrogen at a pressure of $70 \mathrm{MPa}$, and the rest with air at the atmospheric pressure $(0.101325 \mathrm{MPa})$. The initial temperature is $300 \mathrm{~K}$ everywhere inside the domain. The initial contact surface is assumed to be located in the middle of the release tube.

\subsection{Orifices with the constant areas: fixed elliptical and circular orifices}

Circular and elliptical orifices of equal exit area are studied. Therefore, three different areas based on 1 $\mathrm{mm}, 2 \mathrm{~mm}$ and $5 \mathrm{~mm}$ diameters of circular exits are investigated. For each case, two varying elliptical shapes with the aspect ratios (major axis/minor axis) of $\mathrm{AR}=4$ and $\mathrm{AR}=6$ are considered and compared with their comparable circular counterparts $(\mathrm{AR}=1)$. The dimensions of all the test cases are listed in Table 1.

\subsection{Expanding Orifices}

Two cases of the round orifice with initial diameters of $D_{i}=1 \mathrm{~mm}$ and $D_{i}=2 \mathrm{~mm}$ are considered to evaluate the expanding exit and compare the results to the fixed circular opening with the same initial diameter. Note that the entire release tube is expanding, nevertheless, its length remains $2 \mathrm{~mm}$ long. The expansion rate of the circular hole along the radial direction is $0.2 \mathrm{~mm} / \mu \mathrm{s}$. The boundary nodes move along the normal surface direction (radial axis) with the proposed speed based on the spring analogy [13]. The moving region of the computational domain is restricted to the pressurized vessel and the exit hole, so, only the boundary nodes related to this region move.

\subsection{RESULTS}

\subsection{Fixed Elliptic Orifice in Comparison with Circular Orifice}

One of the important parameters to determine the ignition possibility is the pressure expansion at the hydrogen and air contact surface along the jet centerline. The auto-ignition may occur for sufficiently low rates of expansion [6].

Therefore, the centerline pressure versus time on the contact surface for circular and elliptical exits with different diameters is reported up to a time of $10 \mu$ s (Figure $1(\mathrm{a}, \mathrm{b}$ and $\mathrm{c}$ ). It is observed that by maintaining the same effective area and increasing the aspect ratio of the release hole, pressure on the contact surface expands sooner. This behavior can be negligible for the small size holes (Area $=0.8$ $\mathrm{mm}^{2}$ ), while it is important for a larger size orifice (Area $=19.63 \mathrm{~mm}^{2}$ ). In other words, the elliptical jet escaping from a small hole behaves the same as its comparable circular jet.

The release time is the time when the hydrogen-air interface reaches the exit hole $(\mathrm{z}=0)$. To calculate this time, the contact surface location versus time along the centerline for the orifice size of Area=3.14 $\mathrm{mm}^{2}$ is plotted in Figure 1(d). It is evident that the release time corresponding to varying geometries is virtually equal and it is $\mathrm{t}=0.6 \mu \mathrm{s}$. It is noticed in Figure 1(b), when the gas escaping into the air, after a short delay, the interface pressure starts expanding with time.

The change in the orifice geometry affects the development of the jet (Figure 2). Spreading and mixing characteristics differ from elliptical and circular jets. The hydrogen jet releasing from the elliptical hole spreads more quickly in the minor axis plane than the major axis plane and, in turn, it mixes with 
air faster along the minor axis and advances through the ambient air more quickly. Clearly, the circular hydrogen jet spreads with the same rate in both directions and the mixing rate does not change along the minor and major axes. The unequal spreading rates are caused by the non-uniform curvature variation of the elliptic orifice. The higher spreading rate along the minor axis plane results in the axisswitching phenomenon which is recognized in the elliptic jets for both aspect ratios of 4 and 6 and under both pressures of $10 \mathrm{MPa}$ and $70 \mathrm{MPa}$. The iso-surfaces of the Mach number $(\mathrm{M}=2)$ for elliptical and circular jets with the area of $\mathrm{A}=0.8 \mathrm{~mm}^{2}$ are illustrated in Figure 2. In these contours, only the Mach numbers higher than 0.8 are shown and the values less than that are cut off. The crosssectional views of the barrel, high Mach number, and area at different $\mathrm{z}$ locations clearly show the switching of the jet axis. The axis of the jet is switched when the hydrogen comes out of the release exit $(\mathrm{z}=0)$. It is recognized that the hydrogen jet along the minor axis spreads more quickly than the major axis and results in the switching of the jet axis. This behavior is similar for different dimensions and sizes of the elliptical orifice.

The hot temperature of air downstream of the hydrogen-air interface is also one of the important parameters that affect the auto-ignition process [5] [8]. Figure 3 shows the centerline temperature at different times for both circular $(\mathrm{AR}=1)$ and elliptical $(\mathrm{AR}=4)$ orifices with the Area=3.14 $\mathrm{mm}^{2}$. In both cases, temperature has the maximum peak before the hydrogen-air interface reaches the exit $(\mathrm{t}<$ $0.6 \mu \mathrm{s}$ ). As it is shown, the maximum peak take places at $\mathrm{t}=0.2 \mu \mathrm{s}$ and is equal to $3250 \mathrm{~K}$ for both orifices. At the release time $(\mathrm{t}=0.6 \mu \mathrm{s})$ and during the expansion, the highest temperature peak for the elliptic case is slightly lower than its equivalent value in a circular case. For the circular case the maximum temperature decreases from $3150 \mathrm{~K}$ at $\mathrm{t}=0.6 \mu$ s to $3050 \mathrm{~K}$ at $\mathrm{t}=1.0 \mu \mathrm{s}$. For the elliptical case the maximum temperature decreases from $3150 \mathrm{~K}$ at $\mathrm{t}=0.6 \mu \mathrm{s}$ to $2750 \mathrm{~K}$ at $\mathrm{t}=1.0 \mu \mathrm{s}$.

\subsection{Expanding circular release hole in comparison with fixed circular opening}

To evaluate the influence of orifice deformation on the hydrogen jet, an enlarging circular orifice is simulated. The results of expanding circular orifices are compared with the fixed cases with identical initial diameters. Two initial diameters of $1 \mathrm{~mm}$ and $2 \mathrm{~mm}$ with the expanding rate of $200 \mathrm{~m} / \mathrm{s}$ or 0.2 $\mathrm{mm} / \mu \mathrm{s}$ are studied. Two dimensional mesh slices of the expanding release hole with initial diameter of $2 \mathrm{~mm}$ at several times after discharging the hydrogen into air are illustrated in Figure 4.

The location of contact surface during $4 \mu$ s and the pressure versus time for initial diameter of $D_{i}=1$ $\mathrm{mm}$ and $\mathrm{D}_{\mathrm{i}}=2 \mathrm{~mm}$ are compared respectively in Figure 5(a) and Figure 5(b). In all cases, the hydrogen-air interface reaches the exit at $\mathrm{t}=0.6 \mu \mathrm{s}$. It is seen that the contact surface pressures related to expanding release geometries decays sharply in comparison with their fixed counterparts. Note that pressure decay while the interface of the hydrogen-air is still in the tube while the tube is expanding. In addition, the pressure on the interface of hydrogen and air releasing through the smaller area drops more sharply than the larger area exit hole.

The time histories of the contact surface pressure along the centerline (Figure 5(a)) clearly shows that the hydrogen jet issuing from the expanding exit decays sharply and more quickly compared to the fixed circular exit. This behavior starts from $t=0$, that is, before the interface of the hydrogen-air leaves the nozzle. Consequently, the temperature of the hot air downstream of the interface starts decreasing sooner and with a higher rate of decay (Figure 6). It is noticed that during the expansion (at $\mathrm{t}=1 \mu \mathrm{s}$ ), since the contact surface pressure in the enlarging orifice case decays more sharply; a lower temperature peak is observed $(2750 \mathrm{~K})$ compared to the fixed orifice $(3050 \mathrm{~K})$. Therefore, it can be concluded that due to the higher pressure expansion and lower temperature peak, the possibility of auto-ignition for the expanding case is less than the fixed exit case. Another simulation was performed in which the exit was expanded only after the $t=0.6 \mu$ s, i.e. after the contact line reached the exit of the tube [15]. In such a case, the pressure and the temperature behaves the same as for the fixed case. 


\subsection{CONCLUSIONS}

The numerical analysis of hydrogen release from a reservoir at a pressure of $70 \mathrm{MPa}$ shows that the area size of the exit orifice impacts the dispersion and development of a hydrogen jet more than the effect of the orifice shape. Enlarging a circular orifice significantly decreases the pressure at the hydrogen/air interface if the orifice opens when this interface is still in the exit tube. By comparing the hydrogen jet exiting though fixed elliptical and fixed circular orifices of the same area, it is concluded that, as the aspect ratio of the orifice increases, the contact surface pressure decreases and expands more rapidly. Therefore, for an elliptical orifice the contact surface pressure and the peak temperature of the jet decay more quickly than for the case of a jet associated with a comparable circular exit. For the elliptical orifices we also noticed the axis-switching phenomena, which is more dominant for higher reservoir pressures. From this work we conclude that the fixed circular orifice represents a more probable case for auto-ignition in comparison to elliptical orifices having the same area, or to orifices expand in time. Therefore, circular openings represent the most conservative geometry for the study of auto-ignition of hydrogen.

\section{ACKNOWLEDGEMENTS}

This work is supported by the NSERC hydrogen Canada $\left(\mathrm{H}_{2} \mathrm{CAN}\right)$ Strategic Research Network.

\section{References}

[1] Zheng, J.Y., Bie, H.Y., Xu, P., Liu, Y.L., Zhao, Y.Z., Numerical simulation of hydrogen release from high-pressure storage vessel, $3^{\text {rd }}$ International Conference on Hydrogen Safety, Ajacco, France, Sep. 16-18, 2009.

[2] Xiao, J., Travis, J., Breitung, W., Hydrogen release from a high-pressure GH2 reservoir in case of a small leak, 3rd International Conference on Hydrogen Safety, Ajacco, France, Sep. 16-18, 2009.

[3] Khaksarfard, R., Kameshki, M. R. and Paraschivoiu, M., Numerical simulation of high pressure release and dispersion of hydrogen into air with real gas model, Shock Waves, 20, No. 3, 2010, pp. 205-216.

[4] Han, S.H., Chang, D. and Kim, J.S., Release characteristics of highly pressurized hydrogen through a small hole, International Journal of Hydrogen Energy, 38, No. 8, 2013, pp. 3503-3512.

[5] Maxwell, B.M. and Radulescu, M.I., Iignition limits of rapidly expanding diffusion layers: Application to unsteady hydrogen jets, Combustion and Flame Journal, 158, No. 10, 2011, pp. 1946-1959.

[6] Radulescu, M.I., Law, C.K., The transient start of supersonic jets, Journal of Fluid Mechanics, 2007, pp. 331-369. 
[7] Golub, V. V., Baklanov, D. I., Golovastov, S. V., Ivanov, M. F., Laskin, I. N., Saveliev, A.S., Semin, N. V., Volodin, V. V., Mechanisms of high-pressure hydrogen gas self-ignition in tubes, Journal of Loss Prevention in the process industries, 21, 2007, pp. 185-198.

[8] Mogi, T., Kim, D., Shiina, H., Horiguchi, S., Self-ignition and explosion during discharge of highpressure hydrogen, Journal of Loss Prevention in the process industries, 21, No. 2, 2008, pp. 199204.

[9] Yamada, E., Watanaba, S., Hayashi, A. K., Tsuboi, N., Numerical analysis on auto-ignition of a high pressure hydrogen jet spouting from a tube, preceding of $32^{\text {nd }}$ International Combustion Symposium, 32, 2009, pp. 2363-2369.

[10] Golub, V. V., Baklanov, D. I., Bazhenova, T. V., Bragin, M. V., Golovastov, S. V., Ivanov, M. F., Volodin, V. V., Shock-induced ignition of hydrogen gas during accidental or technical opening of high-pressure tanks, Journal of Loss Prevention in the process industries, 20, 2007, pp. 439446.

[11] Xu, B.P., El Hima, L., Wen, J.X., Dembele, S., Tam, V.H.Y. and Donchev, T., Numerical study on the spontaneous ignition of pressurized hydrogen release through a tube into air, Journal of Loss Prevention in the process industries, 21, No. 2, 2008, pp. 205-213.

[12] Wen, J.X., Xu, B.P. and Tam, V.H.Y., Numerical study of spontaneous ignition of pressurized hydrogen release through a length of tube, Combustion and Flame Journal, 156, No. 11, 2009, pp. 2173-2189.

[13] Khaksarfard, R. and Paraschivoiu, M., Numerical simulation of high pressure hydrogen release through an expanding opening, International Journal of Hydrogen Energy, 37, No. 10, 2012, pp. 8734-8743.

[14] Tajallipour, N., Kumar, V., Paraschivoiu, M., Large-eddy simulation of a compressible free jet flow on unstructured elements, International Journal of Numerical Methods for heat \& Fluid, 23, No. 2, 2013, pp. 336-354.

[15] Shishehgaran, N., Numerical Simulation of High Pressure Hydrogen Releases into Air through Varying Orifice Geometries, M. Sc. Thesis, Concordia University, 2013. 


\section{Tables Labels:}

Table 1: Dimensions of different types of orifices

\begin{tabular}{|c|c|c|c|}
\hline \multicolumn{4}{|c|}{$\operatorname{Area}(\mathrm{A})=0.8\left(\mathrm{~mm}^{2}\right)$} \\
\hline Orifice Type & $\begin{array}{l}\text { Major Axis, } \\
\text { a(mm) }\end{array}$ & $\begin{array}{c}\text { Minor Axis, } \\
\text { b(mm) }\end{array}$ & $\begin{array}{c}\text { Aspect Ratio, } \\
\text { a/b }\end{array}$ \\
\hline Circular & 1 & 1 & 1 \\
\hline Elliptical 1 & 2 & 0.5 & 4 \\
\hline Elliptical 2 & 2.45 & 0.41 & 6 \\
\hline \multicolumn{4}{|c|}{$\operatorname{Area}(\mathrm{A})=3.14\left(\mathrm{~mm}^{2}\right)$} \\
\hline Orifice Type & $\begin{array}{c}\text { Major Axis, } \\
\text { a(mm) }\end{array}$ & $\begin{array}{c}\text { Minor Axis, } \\
\mathrm{b}(\mathrm{mm})\end{array}$ & $\begin{array}{c}\text { Aspect Ratio, } \\
\mathrm{a} / \mathrm{b}\end{array}$ \\
\hline Circular & 2 & 2 & 1 \\
\hline Elliptical 1 & 4 & 1 & 4 \\
\hline Elliptical 2 & 5 & 0.82 & 6 \\
\hline \multicolumn{4}{|c|}{$\operatorname{Area}(\mathrm{A})=19.63\left(\mathrm{~mm}^{2}\right)$} \\
\hline Orifice Type & $\begin{array}{c}\text { Major Axis, } \\
\text { a(mm) }\end{array}$ & $\begin{array}{c}\text { Minor Axis, } \\
\text { b(mm) }\end{array}$ & $\begin{array}{c}\text { Aspect Ratio, } \\
\mathrm{a} / \mathrm{b} \\
\end{array}$ \\
\hline Circular & 5 & 5 & 1 \\
\hline Elliptical 1 & 10 & 2.5 & 4 \\
\hline Elliptical 2 & 12.25 & 2.04 & 6 \\
\hline
\end{tabular}



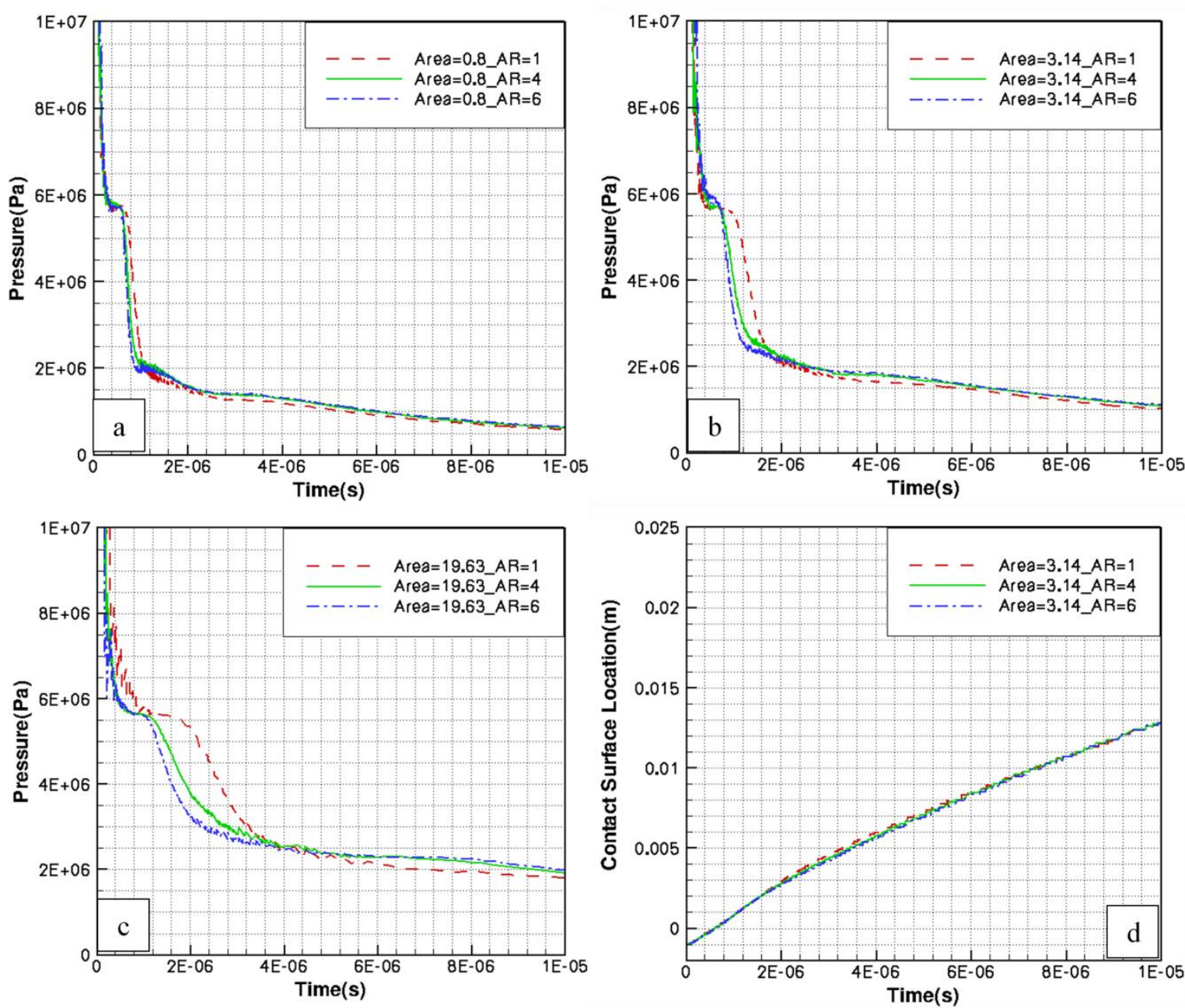

Figure 1: Contact surface pressure versus time for different aspect ratios of release hole, $A R=1, A R=4$ and $\mathrm{AR}=6$ a) Area $=0.8 \mathrm{~mm}^{2}$, b) Area $=3.14 \mathrm{~mm}^{2}$, c) Area $=19.63 \mathrm{~mm}^{2}$, d) the comparison of contact surface location versus time of elliptical and circular orifices (Area=3.14 $\mathrm{mm}^{2}$ ) 


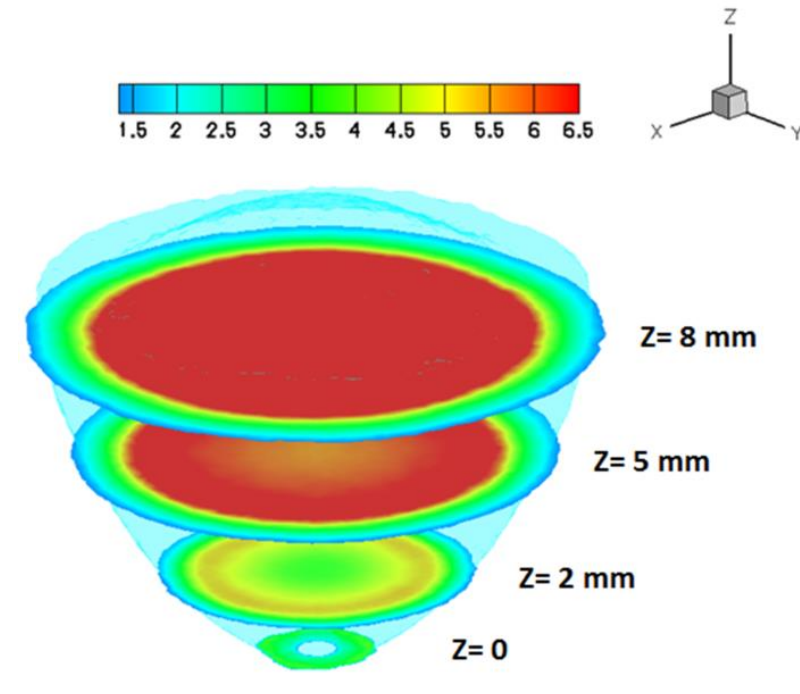

a) $\mathrm{AR}=1$

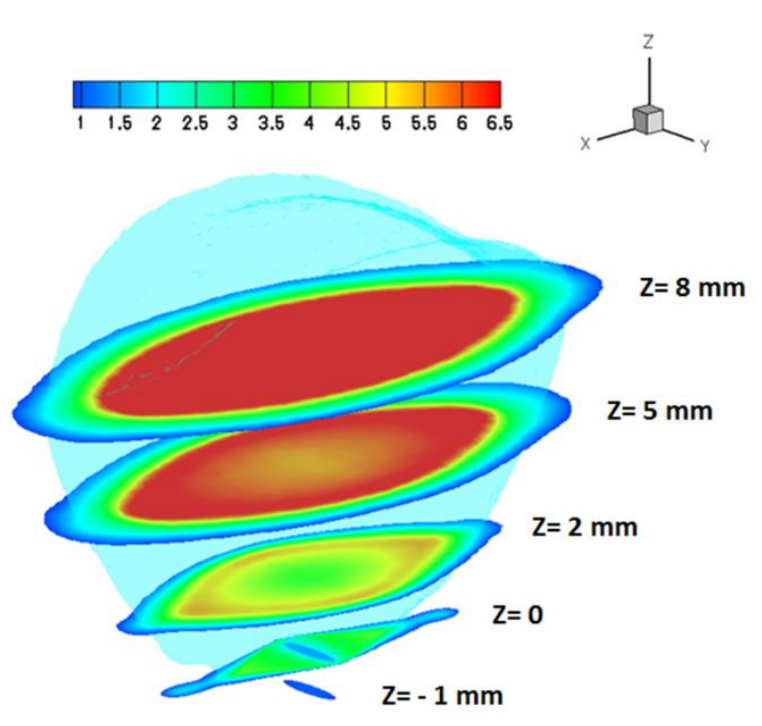

b) $\mathrm{AR}=4$

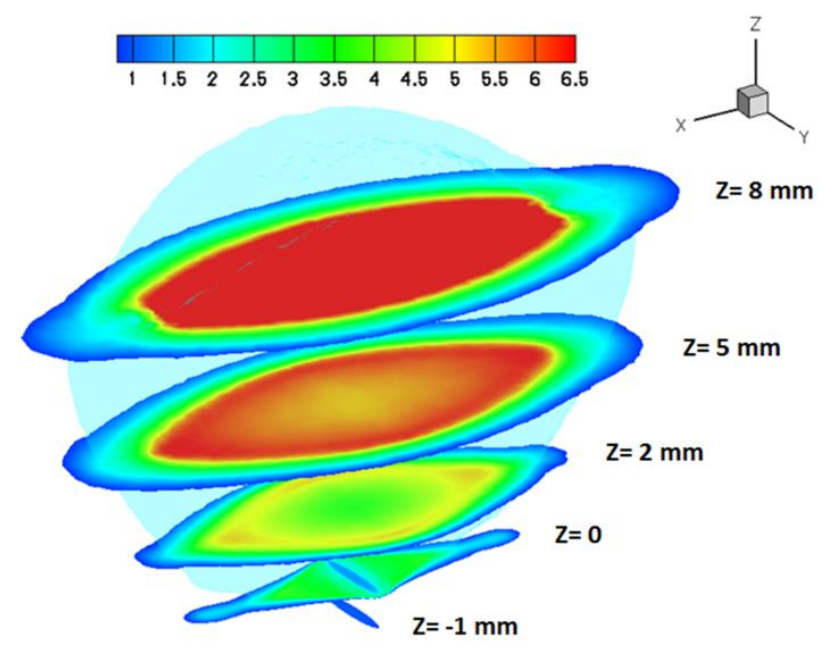

c) $\mathrm{AR}=6$

Figure 2: Iso-surface of Mach number $(\mathrm{M}=2)$ issuing from circular and elliptical orifices, Area $=0.8$ $\mathrm{mm}^{2}, \mathrm{P}=70 \mathrm{MPa}$ a) $\left.\left.\mathrm{AR}=1, \mathrm{~b}\right) \mathrm{AR}=4, \mathrm{c}\right) \mathrm{AR}=6$ 

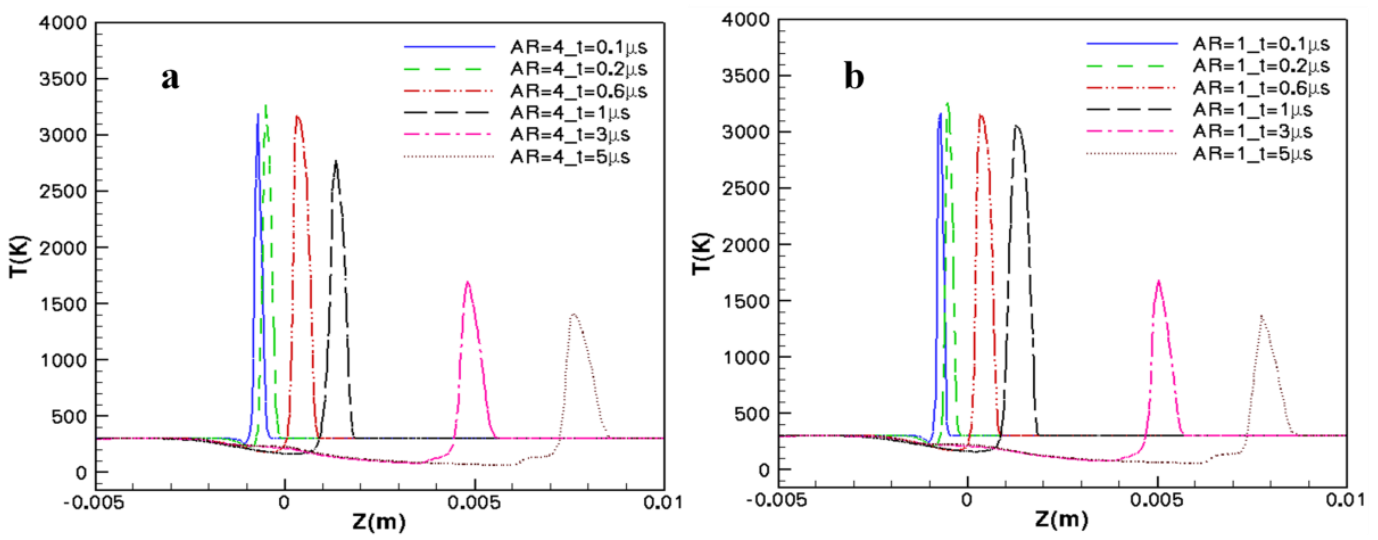

Figure 3: Temperature along the centerline at different times before and after the hydrogen release $\left(\right.$ Area $\left.=3.14 \mathrm{~mm}^{2}\right)$, a) Elliptical orifice b) Circular orifice

a)
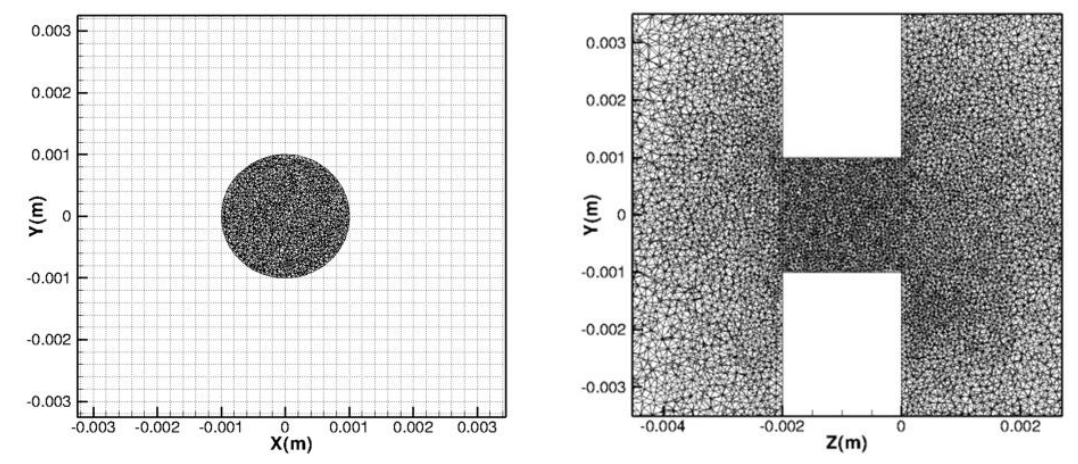

b)
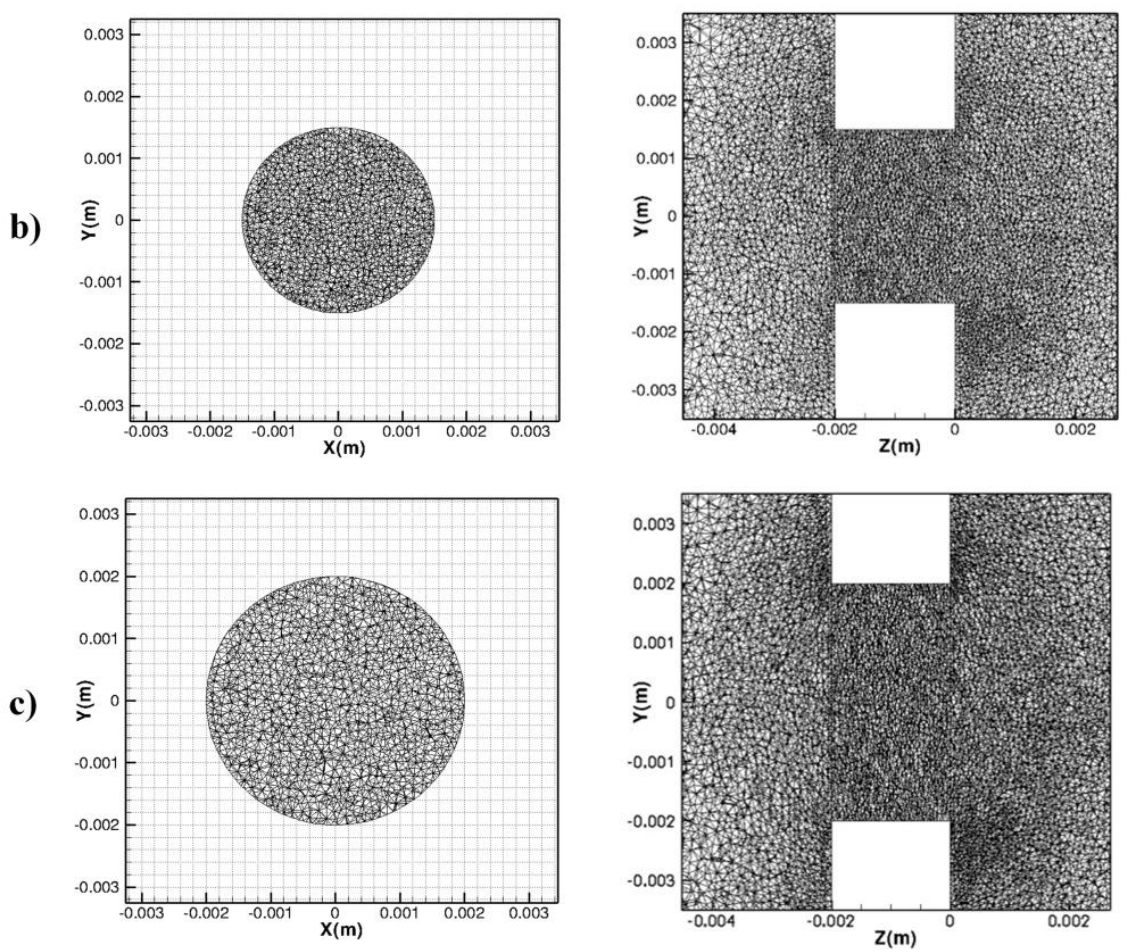

Figure 4: Two dimensional views of the expanding release hole $\left(\mathrm{D}_{\mathrm{i}}=2 \mathrm{~mm}, \mathrm{v}=0.2 \mathrm{~mm} / \mu \mathrm{s}\right), \mathrm{x}-\mathrm{y}$ plane (left), y-z plane (right), a) $\mathrm{t}=0$ (initial diameter), b) $\mathrm{t}=5 \mu \mathrm{s}, \mathrm{c}$ ) $\mathrm{t}=10 \mu \mathrm{s}$ 

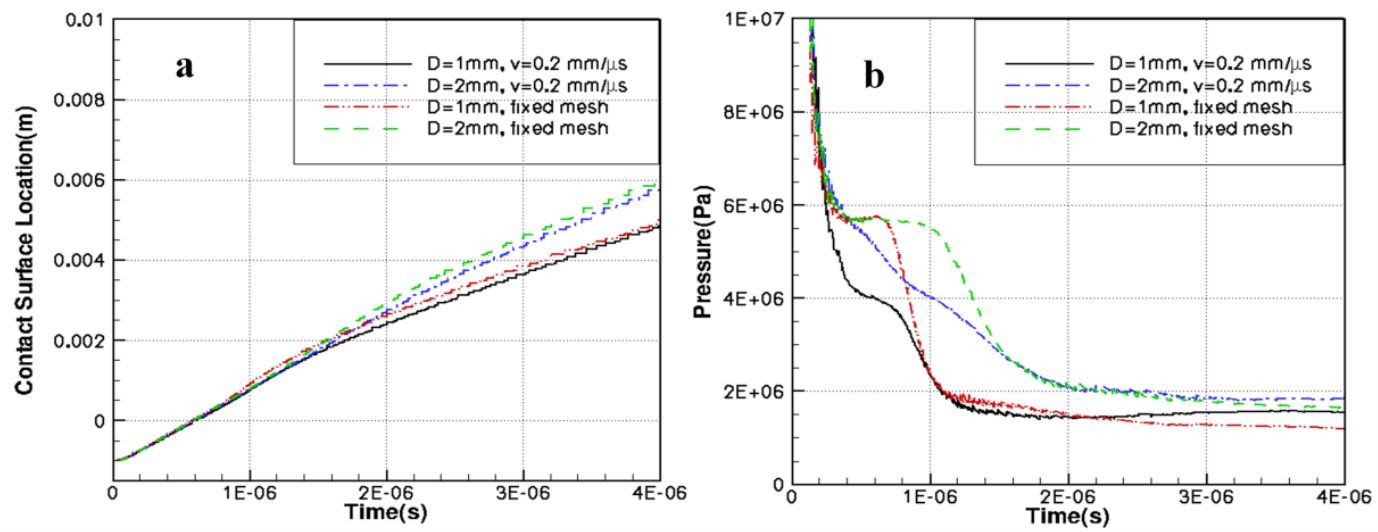

Figure 5: The comparison of a) contact surface location versus time b) Hydrogen-air contact surface pressure versus time of expanding and fixed orifices $\left(D_{i}=1 \mathrm{~mm}\right.$ and $\left.D_{i}=2 \mathrm{~mm}\right)$
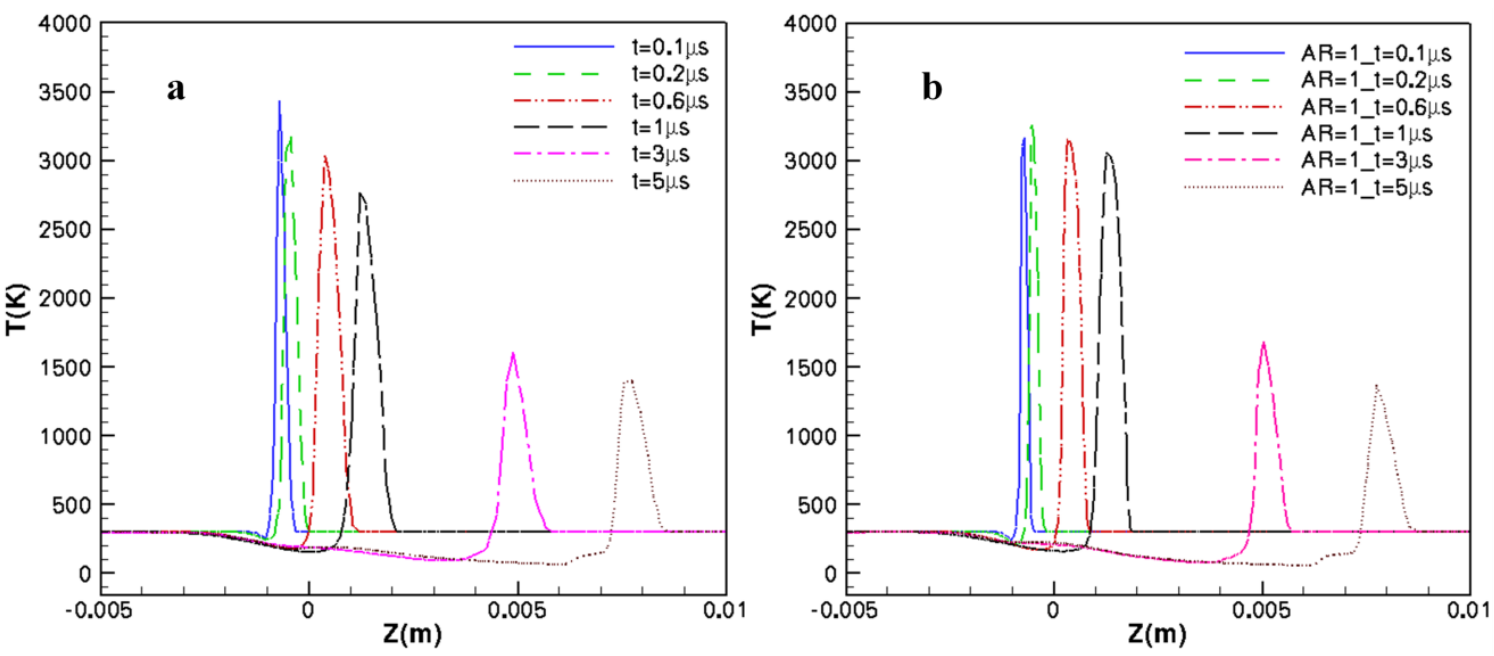

Figure 6: Centerline temperature at several times before and after the hydrogen release $\left(\right.$ Area $\left.=3.14 \mathrm{~mm}^{2}\right)$, a) expanding exit b) fixed circular exit 